\title{
Vulnerable Dispositional Traits and Chronic Pain: Predisposing but not Predetermining
}

Running Title: Vulnerable Dispositional Traits and Chronic Pain

${ }^{1}$ Nicola Sambuco, ${ }^{2,3}$ Angela M Mickle, ${ }^{4}$ Cynthia Garvan, ${ }^{3}$ Josue Cardoso, ${ }^{3}$ Alisa J Johnson, ${ }^{5}$ Daniel A Kusko, ${ }^{5}$ Adriana Addison, ${ }^{6}$ Toni L Glover, ${ }^{3,7}$ Roland Staud, ${ }^{8}$ David Redden, ${ }^{5}$ Burel Goodin, ${ }^{2,3}$ Roger B Fillingim,

${ }^{3,4,9}$ Kimberly T Sibille

${ }^{1}$ College of Public Health and Health Professions, Department of Clinical and Health Psychology, University of Florida, Gainesville, FL

${ }^{2}$ College of Dentistry, Community Dentistry and Behavioral Science, University of Florida, Gainesville, FL ${ }^{3}$ College of Dentistry, Pain Research and Intervention Center of Excellence, University of Florida, Gainesville, FL

${ }^{4}$ College of Medicine, Anesthesiology, University of Florida, Gainesville, FL

${ }^{5}$ College of Arts and Science, Psychology, University of Alabama at Birmingham, Birmingham, AL

${ }^{6}$ School of Nursing, Oakland University, Rochester, MI

${ }^{7}$ College of Medicine, Rheumatology, University of Florida, Gainesville, FL

${ }^{8}$ College of Medicine, Public Health, University of Alabama at Birmingham, Birmingham, AL

${ }^{9}$ College of Medicine, Aging and Geriatric Research, University of Florida, Gainesville, FL

Corresponding Author:

Nicola Sambuco, PhD

Department of Clinical and Health Psychology, University of Florida

PO Box 112766, Gainesville, FL 32611

+1 (352) 3922439

nsambuco@ufl.edu

Declaration of Conflicting Interests: The Authors declare that there is no conflict of interest.

Funding: The authors disclose receipt of the following support for the research, authorship, and publication of this article: This work was supported by the National Institute on Aging R01 AG054370 and R37 AG033906; and National Institute of Mental Health R01 MH112558-01. The content is solely the responsibility of the authors and does not necessarily represent the official views of the National Institutes of Health.

Acknowledgments: The authors would like to thank the UPLOAD-2 participants, the University of Florida Clinical and Translational Science Institute nursing staff and the UPLOAD2 research teams at the University of Florida and University of Alabama at Birmingham. Appreciation is also expressed for the feedback provided by Taylor Buchanan, M.Ed., PHD. 


\begin{abstract}
Dispositional traits can be protective or contribute to increased vulnerability in individuals with chronic pain. This study aims to evaluate the association between two dispositional trait measures, affect balance style and multi-domain trait groups, with psychosocial measures, clinical pain, functional pain, and experimental pain at two years in individuals with chronic knee pain. The study is a prospective analysis of 168 community dwelling individuals aged $45-85$ years old with knee pain with or at risk for knee osteoarthritis. At baseline, affect balance style and multi-domain trait groups were associated with psychosocial measures, clinical pain, and functional status. At the two-year time point, the multi-domain trait groups were associated with the clinical pain measures. Interestingly, individuals with previously demonstrated vulnerable traits showed more variability in dispositional trait status at the two-year time point compared to those with dispositional traits previously demonstrated as more protective. Findings reiterate that dispositional traits are predisposing but are not predetermining regarding pain-related experiences.
\end{abstract}

\title{
Perspective
}

Vulnerable and protective dispositional traits are positively and negatively associated with clinical pain and functional limitations respectively. Although considered relatively stable, a $30-50 \%$ shift in dispositional traits was indicated over a two-year period. Findings suggest dispositional traits may be modifiable and thus, predisposing but not predetermining for persisting chronic pain.

Keywords: Dispositional Traits, Temperament, Affect, Musculoskeletal Pain, Osteoarthritis 


\section{Introduction}

Reactivity to life events, including pain-related experiences, is influenced by dispositional traits. Dispositional traits, also known as temperament, are neurologically-based, supported by an extensive theoretical foundation, and grounded in a strong body of evidence across disciplines and species [1-12]. Highly predictive of behavior, social interactions, physiological reactivity, and responses to stressful events $[11,13,14]$, some dispositional traits are protective while others increase vulnerability for worse psychological and physical health outcomes [15-17].

Affect Balance Style, a measure of dispositional trait affect, has been investigated in crosssectional studies in healthy and chronic pain populations $[18,19]$. Trait positive (PA) and negative (NA) affect are temperamental in nature and associated with positive and negative emotions, respectively [2, $8,20,21]$. The two dimensions are also linked to mood and brain motive systems underlying appetitive and defensive behaviors [21-24]. ABS investigates the combined influence of PA and NA on pain-related measures with findings suggesting the predictive utility extends beyond trait PA or NA alone $[25,26]$. Investigated in adults with fibromyalgia [27, 28] chronic low back pain [18], and in pain-free individuals [19], a consistent pattern demonstrates that Healthy and Low ABS are the more "protective" styles as individuals report lower levels of clinical pain and psychosocial distress contrasted to the more "vulnerable" styles, Depressive and Reactive ABS, where individuals report greater clinical pain and more psychosocial distress [18, 19, 27].

Although affect is recognized as a primary contributor in dispositional traits, other domains of functioning are involved including cognitive, physiological regulation, somatosensory, and sensorimotor $[2,5,7]$. Chess and Thomas [5], developed the criteria to assess temperament in infants, which incorporated domains of functioning including affect/emotional expression, motor, sensory, social, arousal/reactivity, and attentional. Additional evidence aligns with a multi-domain conceptualization [2, 7], suggesting that dispositional traits extend beyond affect. Further, affect is not the only domain showing bidirectional connections with pain. For example, pain and cognition are known to interact reciprocally [29-34]. Additionally, there is evidence of associations between somatosensory sensitivity and chronic pain [35]. The Research Domain Criteria project (RDoC) is a multi-level research framework introduced by the National Institutes of Mental Health (NIMH) to define a new classification system that aims to address limitations in the current category-based diagnostic system [36-38]. Therefore, investigations of associations between chronic pain and a simple measure of dispositional traits based on affect, $A B S$, compared to a more comprehensive measure of multi-domain traits would inform research moving forward.

The purpose of this study was three-fold: 1a) to confirm anticipated ABS group differences in psychosocial, clinical pain, functional status and experimental pain measures; $1 \mathrm{~b}$ ) to assess multidomain trait group differences in the above outcomes of interest; and 2) to assess whether dispositional trait phenotypes measured by ABS and multi-domain trait groups are associated with the outcomes of interest two-years following the baseline time point. Aim 1a represents a replication of earlier studies in a new population $[9,27,28,39]$. Aim $1 \mathrm{~b}$ extends the research by investigating a more comprehensive measure of dispositional traits. Aim 2 represents a new contribution, investigating the longitudinal association between two dispositional trait measures and psychosocial and pain-related outcomes. Findings will help inform whether multi-domain trait groups represent an improved operationalization of dispositional traits than ABS. Also, if, as suggested by studies reporting high temporal stability of trait 
characteristics [6], dispositional characteristics are predetermining, then longitudinal analyses are expected to demonstrate a stable association between trait measures and psychosocial/pain outcomes. If, however, dispositional characteristics are only predisposing factors, then poor associations are expected between dispositional traits at baseline and psychosocial/pain outcomes at two-years followup, indicating the possibility to modify trait characteristics.

\section{Methods}

\section{Participants}

The current study is a prospective analysis. Procedures described are limited to the present study. The STROBE cross-sectional checklist was used in reporting [40]. Adults between 45 and 85 years of age who self-identified as non-Hispanic Black or Non-Hispanic White with knee pain with or at risk for knee osteoarthritis were recruited and enrolled in the Understanding Pain and Limitations in Osteoarthritic Disease-Two (UPLOAD-II) study at the University of Florida and University of Alabama at Birmingham. Participants were recruited through multiple community advertisement methods. Study sessions were conducted between June 2015 and July 2019. Inclusion and exclusion criteria have been previously reported [41]. Participants who completed the Positive and Negative Affect Schedule questionnaire and reported knee pain based on a validated osteoarthritis screening questionnaire [42] were included. The UPLOAD-II study was approved by both the University of Florida and the University of Alabama at Birmingham Institution Review Boards. All participants provided verbal and written informed consent.

\section{Procedures}

Participants completed three study sessions at baseline. Data for the present study were collected during two of the three sessions. The first session was a health assessment where participants completed questionnaires regarding sociodemographics, health status, history of clinical pain, cognitive assessment, psychosocial measures, and height and weight were collected, and body mass index (BMI) calculated. At the second session, participants completed quantitative sensory testing (QST). Approximately two years after the baseline sessions, participants returned and repeated the same study procedures. Between the baseline and two year follow up visits, participants also completed quarterly health assessments with information collected specific to clinical interventions (surgery, other procedures, or new treatments for pain).

\section{Predictive measures}

Sociodemographic and Health Status. Sociodemographic characteristics collected include age, sex, self-reported ethnicity/race, and highest education. Participants completed a health questionnaire assessing comorbidities, including high blood pressure, heart disease, cancer, diabetes, asthma/breathing problems, kidney disease, thyroid problem, stroke, seizure, chronic pain, neurological disorder, depression, and other mental health conditions or other health problem.

Affect Balance Style. Positive and Negative Affect Schedule (PANAS)[8]. The PANAS consists of ten positive words and ten negative words comprised of emotions and feelings. Scores range from 10-50 for both positive (PA) and negative (NA) affect, with higher scores indicating higher levels. Consistent with prior studies [19], participants were categorized into one of four ABS groups based on published 
adult normative means for the PANAS: Healthy: high PA >35 and low NA $\leq 18.1$; Low: low PA $\leq 35$ low NA $\leq 18.1$; Reactive: high PA >35 high NA >18.1, Depressive: low positive PA $\leq 35$ and high NA >18.1.

Multi-Domain Trait Groups. The RDoC matrix contains six domains including: 1) positive and 2) negative valence, 3) cognitive systems, 4) social processes, 5) arousal and regulatory systems, and 6) sensorimotor systems. Positive and negative valence refers to an individual's responses to experiences in a rewarding/positive or aversive/negative situations. Cognitive systems are measures of cognitive processes such as attention, memory, perception, and cognitive control. Social processes refer to perception and interpretation of others' action in interpersonal settings. Arousal and regulatory systems initiate the activation of neural systems and provides homeostatic regulation of such systems, while the sensorimotor systems assess control and execution of motor actions. Based on the identified constructs and elements within each of the six domains for the RDoC [43], available measures within the current dataset were reviewed with a goal of identifying a minimum of two measures per domain. This criterion was based on the fact that RDoC domains are operationalized as broad constructs that extend beyond measurements that can be captured by a single questionnaire or measure.

\section{Negative Valence:}

Positive and Negative Affect Schedule (PANAS) [1]. The PANAS negative scale was used for this analysis as described above.

PROMIS-Anxiety 7a [2]. The PROMIS-anxiety measures emotional distress in the past 7 days on a 5-point Likert scale from 1-never to 5-always. Raw scores were calculated then normalized against Tscores. Higher scores indicating greater anxiety.

\section{Positive Valence:}

Positive and Negative Affect Schedule (PANAS) [1]. The PANAS positive scale was used for this analysis as described above.

Life Orientation Test-Revised (LOT-R)[3]. The LOT-R is an instrument to measure the level of optimism such as happiness, motivation, and self-confidence. Each statement is assessed on a 5-point Likert scale from 0-strongly disagree to 4-strongly agree. Scores range from 0-24, with low scores 0-13 indicating low optimism, 14-18 moderate optimism, and 19-24 indicating high optimism.

\section{Cognitive:}

Montreal Cognitive Assessment (MOCA)[4]. The MOCA is a brief cognitive assessment consisting of a memory recall, clock drawing, cube copy, trail making B, phonemic fluency, verbal abstraction, naming, complex sentence, and fluency task

Rapid Estimate of Adult Literacy in Medicine, Revised (REALM-R)[5]. The REALM-R assess literacy. Participants read a series of 11 words out loud. A score of 6 or less indicates low health literacy. Of note, in individuals with a score of 6 or less, study staff read out loud and completed all questionnaires with the participant to ensure comprehension and completion of the assessments.

\section{Arousal and Regulatory:}


Fatigue Severity Scale (FSS)[6]. The FSS is a 9-item scale that measures fatigue and the effects on daily activities. Statements are assessed within the past week on a 7-point Likert scale from 1-strongly disagree to 7-strongly agree. Scores range from 9-63, with higher scores indicating greater fatigue.

Blood Pressure. Blood pressure was measured four continuous times before QST testing. The first measurement was dropped, and the average of all three remaining measurements was used in the analysis.

\section{Sensorimotor:}

PainDETECT Questionnaire (PD-Q)[7]. The PD-Q assesses neuropathic pain of the knee. Questions include whether the pain is persistent or intermitting and include symptoms such as burning, prickly or numbing sensations. Scores range from -1 to 38 with higher scores indicating a neuropathic component of pain.

Warmth Detection. Thermal stimuli were delivered using Medoc Thermal Sensory Analyzer (Ramat Yishai, Israel) to the most painful knee and ipsilateral forearm. Warm threshold was assessed by the gradual increase of heat until the participant indicated they could first feel the warmth. Testing was conducted three times at each site (knee and arm), and means calculated for each of the three thermal tests.

\section{Social:}

Only one measure reflecting one of the elements of social processes was available in the current dataset, the Multidimensional Scale of Perceived Social Support [44]. However, since the social domain includes several constructs (affiliation and attachment, social communication, perception and understanding of self, perception, and understanding of others) and each of them is further divided into many subconstructs, we reasoned that a single measure was not sufficient to comprehensively describe and represent this complex domain. Therefore, the MSPSS was not included in the principal component analysis.

\section{Outcome Measures}

\section{Psychosocial Measure.}

Patient-Reported Outcomes Measurement Information Symptom Measures System (PROMIS) Depression (SF8b)[45]. The PROMIS depression scale consists of 8 items on a 5-point Likert scale ranging from 1- never to 5-always. Raw scores were calculated then normalized against T-scores. Higher scores are consistent with increased depression.

\section{Clinical Pain Measures.}

The Western Ontario and McMaster Universities Osteoarthritis Index (WOMAC)[46, 47]. The WOMAC evaluates knee osteoarthritis and consists of 24 items divided into three subscales: pain, stiffness, and function. Scores range from 0-20 for pain, 0-8 for stiffness, 0-68 for physical function, and 0-96 total score. Higher scores indicate worse pain, stiffness, and functional limitations.

Graded Chronic Pain Scale (GCPS)[48]. The GCPS assesses the severity of knee pain and its impact on activities on two subscales, characteristic pain intensity (CPI) and disability score over the 
previous six months. The scores range from 0-100, with higher scores indicating greater pain intensity and greater physical disability.

\section{Functional Measures.}

Short Physical Performance Battery (SPPB)[49]. The SPPB assesses functional moving, including a standing balance, 4-meter gait speed, and chair rising task. Scores range from 0-12, with higher scores indicating greater functional ability.

Movement Evoked Pain (MEP)[50]. After each of the tasks of the SPPB, participants are asked to provide a numerical pain rating for their knee using a 0-100 visual analog scale where $0=$ no pain and $100=$ the most pain imaginable. Pain scores are averaged across all tasks, with higher scores indicating greater pain.

\section{Experimental Pain Measures.}

Pressure Pain Threshold. Pressure pain testing was assessed at four sites: medial and ipsilateral knee, trapezius muscle, and quadriceps muscle. The pressure was applied using a hand-held algometer at a constant rate of pressure of $1 \mathrm{~kg} / \mathrm{sec}$. Participants were instructed to indicate when the sensation first became painful and the pressure was recorded. Pressure site and order of experimental pain testing were randomized. Z-scores were calculated for pressure pain at all sites and used in the analysis.

Punctate Pain Testing. Punctate mechanical pain testing was conducted on the most painful knee and the back of the hand using a 300g nylon monofilament. One stimulus then a series of 10 repeated stimuli was delivered twice and participants were asked to report the intensity of pain experienced on a 0-100 scale. Differences in pain ratings of the series and single stimuli were calculated then a z-score calculated over all testing sites. Punctate site and order of experimental pain testing were randomized.

Thermal Pain Testing. Thermal stimuli were delivered using Medoc Thermal Sensory Analyzer (Ramat Yishai, Israel) to the most painful knee and ipsilateral forearm. Thermal pain threshold was assessed by gradual increase of heat until the participant indicated the first sensation of pain. Thermal pain tolerance was assessed by the gradual increase of heat until the participant indicated the pain was no longer tolerated. Testing was conducted three times at each site (knee and arm) and means calculated for each of the three thermal tests. Z-scores were then calculated, including all testing sites, both threshold and tolerance heat tests.

\section{Statistical Analysis}

Multi-Domain Trait Group Development. When too many (redundant) predictors are used in a model, over-fitting, generalization, and replication problems arise. For this reason, data dimension reduction through principal component analysis (PCA) has been demonstrated to improve the classification accuracy of taxometric methods such as clustering [51]. The PCA was performed on the correlation matrix after standardizing each measure across the entire sample, and direct oblimin rotation was used that permits correlation between components. The PCA analysis resulted in five factors with eigenvalues greater than one (explaining $71.6 \%$ of the variance in total), including a factor of negative valence (24.9\% of the variance; high loadings for PANAS-NA and PROMIS-Anxiety), arousal (15.9\% of the variance; high loadings for systolic and diastolic blood pressure), cognitive system (11.9\% 
of the variance; high loadings for MoCA Total Score and REALM-R), positive valence (10.5 of variance; high loadings for PANAS-PA and LOT-R), and sensorimotor system ( $8.4 \%$ of the variance; high loadings for warmth detection-arm and warmth detection-knee). The factor loadings for individual measures are presented in Table S1. The only two correlated factors were negative and positive systems $(r=-.29, p<$ 0.001; the complete correlation matrix is reported in Table S2.

The five components extracted from the PCA were used to cluster individuals in different multidomain groups. The k-means clustering method used in the current analyses is a data-driven multivariate technique where cluster finds partitions defined by centroids, in which the sum of the squared Euclidean distance (the metrics chose for the current analysis) of all cases from assigned cluster centroids is minimized. Since k-means clustering is an unsupervised technique in which the only constraint is represented by the number of pre-specified clusters ( $k$ ), the optimal number of $k$ and the corresponding classification was assessed using the Silhouette coefficient method [52] for a number of clusters solution ranging from 2 to 10 . The highest silhouette coefficient value was found for the twocluster solution (silhouette values for different levels of $\mathrm{k}: 2=.34,3=.31,4=.33,5=.30,6=.29,7=$ $.27,8=.27,9=.30,10=.31$ ), that represents the most appropriate to describe the underlying structure of the data. The nature of the differences between clusters was then examined in a series of independent samples t-tests using cluster as a between-subjects factor (cluster 1: $N=106$; cluster 2: $N=$ 61 ) and each of the factor scores from the PCA as a within subject factors. As Figure 1 illustrates, participants in cluster 1 - compared to cluster 2 - had a lower score at the negative system $\left(t_{165}=-10.32, p<0.001\right.$, Cohen's $d=-1.66)$ and the sensorimotor system $\left(t_{165}=-3.09, p=0.003\right.$, Cohen's $d=-.49$ ), with a higher score found for the cognitive system ( $t_{165}=7.04, p<0.001$, Cohen's $d=1.13$ ) and positive system ( $t_{165}=8.07, p<0.001$, Cohen's $\left.d=1.30\right)$; no group differences were detected for the arousal system $\left(t_{165}=-.57, p\right.$ $=0.57)$. Differences between the clusters for the measures used in the PCA are reported in Table S3. Given the pattern of differences that characterize the two clusters, cluster 1 was named Positive Valence and Cognition and cluster 2 was named Negative Valence and Sensorimotor (Figure 1).

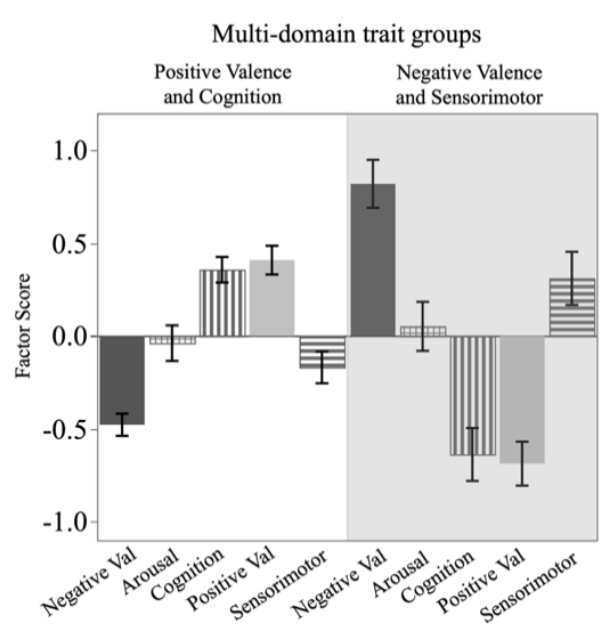

Figure 1. The underlying structure of the two multi-domain trait groups solution with k-means cluster revealed that a group of Positive Valence and Cognition (left) characterized by low scores on negative valence, arousal, and sensorimotor with a concurrent high score for positive valence and cognition, while the opposite pattern characterizes the Negative Valence and Sensorimotor (right).

Analytic Strategy. To test Aims 1, regression analyses were performed using groups (ABS or multi-domain traits) as between-subject factors and adjusting for sex, age, ethnicity/race, the total number of pain sites, income, and BMI on outcomes of interest including psychosocial measure, clinical pain, functional status and experimental pain. To test Aim 2, longitudinal regression analyses adjusting for sex, age, ethnicity/race, the total number of pain sites, income, and BMI were conducted on psychosocial measure, clinical pain, functional status and experimental pain at the two-year time point, 
further adjusting the baseline level of outcome variables. An additional post-hoc analysis was conducted also adjusting for whether the participants reported new pain treatment anytime on the quarterly health surveys during the two-years period. When significant differences in ABS groups were detected in the regression models for Aim 1 and Aim 2, follow-up one-way ANOVAs were conducted adjusting the $p$ values for a family of three comparisons using Tukey HSD. When groups differences between multidomain groups were detected in the regression models for Aim 1 and Aim 2, follow-up analyses were conducted using independent sample t-tests. To adequately control for inflation of Type I error due to multiple hypothesis testing, false discovery rate (FDR) using the Benjamini-Hochberg procedure was calculated for both Aim 1 and Aim 2, with results considered significant at FDR $<0.05$. 


\begin{tabular}{|c|c|c|c|c|c|c|c|c|c|}
\hline \multirow[t]{2}{*}{ Variable } & \multicolumn{5}{|c|}{ ABS groups } & \multicolumn{4}{|c|}{ Multi-domain trait groups } \\
\hline & $\begin{array}{l}\text { Total } \\
(\mathrm{N}=168)\end{array}$ & $\begin{array}{l}\text { Healthy } \\
(\mathrm{N}=69)\end{array}$ & $\begin{array}{l}\text { Low } \\
(n=49)\end{array}$ & $\begin{array}{l}\text { Reactive/Depressive } \\
\qquad(\mathrm{n}=50)\end{array}$ & $p$-value & $\begin{array}{l}\text { Total } \\
(\mathrm{N}=167)\end{array}$ & $\begin{array}{l}\text { Positive Valence and } \\
\text { Cognition } \\
(\mathrm{N}=106)\end{array}$ & $\begin{array}{l}\text { Negative Valence } \\
\text { and Sensorimotor } \\
\qquad(\mathrm{n}=61)\end{array}$ & $p$-value \\
\hline Age, median [IQR] & $57.0[11.0]$ & $60.0[10.0]$ & $58.0[15.0]$ & $53.0[6.0]$ & $<0.0001$ & $57.0[11.0]$ & $58.5[12.0]$ & $55.0[7.0]$ & 0.0009 \\
\hline Gender, N (\%) & & & & & 0.7365 & & & & 0.1164 \\
\hline Male & $61(36.3)$ & $24(34.8)$ & $20(40.8)$ & $17(34.0)$ & & $61(36.5)$ & $34(32.1)$ & $27(44.3)$ & \\
\hline Female & $107(63.7)$ & $45(65.2)$ & $29(59.2)$ & $33(66.0)$ & & $106(63.5)$ & 72 (67.9) & $34(55.7)$ & \\
\hline Race/Ethnicity, N (\%) & & & & & 0.2723 & & & & 0.0041 \\
\hline Non-Hispanic Black & $83(49.4)$ & $33(47.8)$ & $22(44.9)$ & $30(60.0)$ & & $85(50.9)$ & $45(42.5)$ & $40(65.6)$ & \\
\hline Non-Hispanic White & $85(50.6)$ & $36(52.2)$ & $27(55.1)$ & $20(40.0)$ & & $82(49.1)$ & $61(57.6)$ & $21(34.4)$ & \\
\hline Body Mass Index, median [IQR] & $31.4[10.1]$ & $30.3[8.3]$ & $31.6[10.5]$ & $31.8[11.5]$ & 0.5455 & $31.4[10.1]$ & $30.5[10.1]$ & $32.3[8.1]$ & 0.0833 \\
\hline No. Pain Sites, median [IQR] & $5.0[4.5]$ & $5.0[4.0]$ & $5.0[4.0]$ & $6.0[4.0]$ & 0.6672 & $5.0[4.0]$ & $5.0[4.0]$ & $6.0[5.0]$ & 0.0065 \\
\hline Current Comorbidities (0-14) , N (\%) & & & & & 0.4052 & & & & 0.0125 \\
\hline $0-1$ & $105(62.5)$ & $49(71.0)$ & $29(59.2)$ & $27(54.0)$ & & $105(37.1)$ & $76(71.7)$ & $29(47.5)$ & \\
\hline $2-3$ & $52(31.0)$ & $17(24.6)$ & $19(38.8)$ & $16(32.0)$ & & $51(30.5)$ & $27(25.5)$ & $24(39.3)$ & \\
\hline $4-7$ & $11(6.5)$ & $3(4.3)$ & $1(2.0)$ & $7(14.0)$ & & $11(6.6)$ & $3(2.8)$ & $8(13.1)$ & \\
\hline Education, $\mathbf{N}(\%)$ & & & & & 0.0033 & & & & $<0.0001$ \\
\hline High school or less & $76(45.2)$ & $24(34.8)$ & $19(38.8)$ & $33(66.0)$ & & $76(45.5)$ & $36(34.0)$ & $40(65.6)$ & \\
\hline Higher education & $92(54.8)$ & $35(50.7)$ & $30(61.2)$ & $17(34.0)$ & & $91(54.5)$ & $70(66.0)$ & $21(34.4)$ & \\
\hline Income, $\mathrm{N}(\%)$ & & & & & 0.0009 & & & & \\
\hline$\$ 0-29,999$ & $92(54.8)$ & $29(42.0)$ & $23(46.9)$ & $40(80.0)$ & & $91(54.5)$ & $42(39.6)$ & $49(80.3)$ & $<0.0001$ \\
\hline$\$ 30,000-79,999$ & $49(29.2)$ & $25(36.2)$ & $17(34.7)$ & $7(14.0)$ & & $49(29.3)$ & $40(37.7)$ & $9(14.8)$ & \\
\hline$\$ 80,000+$ & $26(15.5)$ & $11(15.9)$ & $9(18.4)$ & $3(6.0)$ & & $26(15.6)$ & $23(21.7)$ & $3(4.9)$ & \\
\hline Not Reported & $1(0.6)$ & $1(1.4)$ & $0(0.0)$ & $0(0.0)$ & & $1(0.6)$ & $1(0.9)$ & $0(0.0)$ & \\
\hline Study Site, N (\%) & & & & & 0.6872 & & & & 0.4117 \\
\hline UF & $108(64.3)$ & 47 (68.1) & $30(61.2)$ & $31(62.0)$ & & $108(64.7)$ & $71(67.0)$ & $37(60.7)$ & \\
\hline UAB & $60(35.7)$ & 22 (31.9) & $19(38.8)$ & $19(38.0)$ & & $59(35.3)$ & $35(33.0)$ & $24(39.3)$ & \\
\hline
\end{tabular}

Table 1: Baseline characteristics of participants by ABS groups and multi-domain trait groups. 


\section{Results}

\section{General Descriptives}

A total of 168 participants self-reported knee pain and completed the PANAS questionnaires and 167 participants had complete data for the multi-domain trait analyses. Baseline characteristics of participants by both ABS and multi-domain trait groups are presented in Table 1. Income and education were significantly different between ABS groups. There were a number of significant differences between multi-domain trait groups, including age, ethnicity/race, number of pain sites, number of comorbidities, education, and income.

\section{ABS Groups on Psychosocial, Clinical and Experimental Pain Measures}

In multiple regression analysis adjusting for sociodemographic characteristics (age, sex, ethnicity/race, number of pain sites, income, and BMI), ABS groups was associated with depressive symptomatology (PROMIS depression), clinical pain (WOMAC total), and functional status (SPPB) (Table 2). Follow-up one-way ANOVAs with planned comparisons revealed that depressive/reactive individuals reported higher levels of depression ( $M=56.4 ; S D=7.5)$ and clinical pain $(M=45.7 ; S D=19.7)$ than both healthy (PROMIS depression: $\mathrm{M}=43.8, \mathrm{SD}=6, \mathrm{t}=9.37, \mathrm{p}<.001$; WOMAC total: $\mathrm{M}=30.7, \mathrm{SD}=18.3, \mathrm{t}=4.25$, $\mathrm{p}<.001$ ) and low (PROMIS depression: $\mathrm{M}=45.4, \mathrm{SD}=8.3, \mathrm{t}=7.55, \mathrm{p}<.001$; WOMAC total: $\mathrm{M}=34.3, \mathrm{SD}=$ $19, \mathrm{t}=3.01, \mathrm{p}=.008$ ) individuals, with healthy and low individuals did not differ in either depression $(\mathrm{t}=$ $1.18, p=.47)$ nor clinical pain $(t=1, p=.58)$. Depressive individuals additionally reported higher levels of functional pain than healthy individuals (depressive: $M=1.73, S D=4$; healthy: $M=1.47, S D=6 ; t=3.01, p=$ $.008)$.

\begin{tabular}{|c|c|c|c|c|c|c|}
\hline & \multicolumn{3}{|c|}{ ABS Groups } & \multicolumn{3}{|c|}{ Multi-domain trait groups } \\
\hline & $\mathrm{N}$ & F-Value & P-Value & $\mathrm{N}$ & F-Value & P-Value \\
\hline \multicolumn{7}{|l|}{ Psychosocial } \\
\hline PROMIS Depression & 166 & 31.37 & $<.0001 *$ & 165 & 35.21 & $<0.0001^{*}$ \\
\hline \multicolumn{7}{|l|}{ Clinical Pain } \\
\hline WOMAC total & 164 & 23.15 & $<.0001 *$ & 163 & 29.38 & $<0.0001 *$ \\
\hline GCPS CPI & 166 & 0.84 & 0.4348 & 165 & 12.26 & $0.0006^{*}$ \\
\hline GCPS disability & 166 & 1.12 & 0.3280 & 165 & 8.04 & $0.0052 *$ \\
\hline \multicolumn{7}{|l|}{ Functional Pain } \\
\hline SPPB & 166 & 3.82 & 0.0240 & 165 & 3.24 & 0.0738 \\
\hline MEP & 165 & 2.35 & 0.0990 & 164 & 14.46 & $0.0002 *$ \\
\hline \multicolumn{7}{|l|}{ Experimental Pain } \\
\hline Punctate & 166 & 1.92 & 0.1493 & 165 & 3.60 & 0.0595 \\
\hline Heat & 166 & 0.42 & 0.6578 & 165 & 0.21 & 0.6470 \\
\hline Pressure & 165 & 0.37 & 0.6936 & 165 & 2.76 & 0.0988 \\
\hline
\end{tabular}

Table 2. Group differences at baseline assessment for ABS groups and multi-domain trait groups on psychosocial measures, clinical pain, functional status and experimental pain measures.

Comparisons include sex, age, ethnicity/race, total number of pain sites, body mass index, and income as covariates. Note. ${ }^{*}$ FDR (false-discovery rate) $<0.05$. WOMAC: Western Ontario and McMaster Universities Osteoarthritis Scale, GCPS CPI: Graded Chronic Pain Scale Characteristic Pain Intensity, GCPS: Graded Chronic Pain Scale, SPPB: Short Physical Performance Battery, MEP: Movement Evoked Pain 


\section{Multi-Domain Trait Groups on Psychosocial, Clinical and Experimental Pain Measures}

In multiple regression analysis using the same predictors and adjusting for sociodemographic and health characteristics, the multi-domain trait groups were predictive of depressive symptomatology (PROMIS depression), clinical pain measures (WOMAC total, GCPS CPI, GCPS disability), and functional status pain (MEP) (Table 2). Independent sample t-tests revealed that individuals in the Negative Valence and Sensorimotor cluster, compared to the individuals in the Positive Valence and Cognition cluster, reported higher levels of depression ( $M=54.1$ vs. $M=44.4, t_{165}=7.84, p<.001$, Cohen's $d=1.26$ ), clinical pain (WOMAC total: $\mathrm{M}=50$ vs. $\mathrm{M}=28, \mathrm{t}_{163}=8.13$, $\mathrm{p}<.001$, Cohen's $d=1.31 ; \mathrm{GCPS} C P I$ : $\mathrm{M}=69.1$ vs. $M=47.2 ; t_{165}=6.57, p<.001$, Cohen's $d=1.06$; GCPS disability: $M=62.6$ vs. $M=38.2 ; t_{165}=5.34, p<.001$, Cohen's $d=.86)$, and functional pain ( $M=35.8$ vs. $M=15.1, \mathrm{t}_{164}=5.35, \mathrm{p}<.001$, Cohen's $\left.d=.86\right)$.

In line with findings from ABS groups, no differences in experimental pain measures were detected between the two groups.

\section{Associations between ABS Groups and Health Outcomes at 2 Years}

When adjusting for the baseline sociodemographic characteristics and baseline measure, ABS groups were not predictive of any measure at two years following baseline (Table 3 ). Identical results were obtained when information about new treatment or surgery, done in the two years period between the baseline and the follow-up assessment, were included in the multiple regression.

\begin{tabular}{|c|c|c|c|c|c|c|}
\hline & \multicolumn{3}{|c|}{ ABS groups } & \multicolumn{3}{|c|}{ Multi-domain trait groups } \\
\hline & $\mathrm{N}$ & F-Value & P-Value & $\mathrm{N}$ & F-Value & P-Value \\
\hline \multicolumn{7}{|l|}{ Psychosocial } \\
\hline PROMIS Depression & 115 & 0.84 & 0.4367 & 114 & 0.08 & 0.7740 \\
\hline \multicolumn{7}{|l|}{ Clinical Pain } \\
\hline WOMAC total & 121 & 0.42 & 0.6607 & 120 & 7.38 & $0.0077^{*}$ \\
\hline GCPS CPI & 127 & 0.02 & 0.9787 & 126 & 8.11 & $0.0052 *$ \\
\hline GCPS disability & 127 & 0.18 & 0.8317 & 126 & 4.81 & 0.0303 \\
\hline \multicolumn{7}{|l|}{ Functional Pain } \\
\hline SPPB & 125 & 0.15 & 0.8635 & 124 & 1.21 & 0.2735 \\
\hline MEP & 123 & 1.48 & 0.2320 & 121 & 0.18 & 0.6751 \\
\hline \multicolumn{7}{|l|}{ Experimental Pain } \\
\hline Punctate & 120 & 2.55 & 0.0829 & 119 & 0.06 & 0.8059 \\
\hline Heat & 121 & 1.07 & 0.3481 & 120 & 0.05 & 0.8298 \\
\hline Pressure & 121 & 1.25 & 0.2893 & 121 & 0.00 & 0.9832 \\
\hline
\end{tabular}

Table 3. Group differences at two-years follow-up assessment for ABS and multi-domain trait groups on psychosocial measures, clinical pain, functional status and experimental pain measures. Comparisons include sex, age, ethnicity/race, total number of pain sites, BMI, and income and baseline measure as covariates. Note. * FDR (false-discovery rate) < 0.05. WOMAC: Western Ontario and McMaster Universities Osteoarthritis Scale, GCPS CPI: Graded Chronic Pain Scale Characteristic Pain Intensity, GCPS: Graded Chronic Pain Scale, SPPB: Short Physical Performance Battery, MEP: Movement Evoked Pain

\section{Associations between Multi-Domain Trait Groups and Health Outcomes at 2 Years}

When adjusting for the baseline sociodemographic and health characteristics and baseline measure, multi-domain trait groups were predictive of clinical pain measures (Table 3 ). Independent 
sample t-tests revealed that individuals in the Negative Valence and Sensorimotor cluster, compared to the individuals in the Positive Valence and Cognition cluster, reported higher scores at the WOMAC total $\left(M=40.3\right.$ vs. $M=21.2, t_{93}=5.62, p<.001$, Cohen's $\left.d=1.16\right), G C P S C P I\left(M=58.3\right.$ vs. $M=40.2, t_{96}=4.08, p<.001$, Cohen's $d=.83$ ), and GCPS disability ( $M=47.7$ vs. $M=20.4, t_{96}=5.64, p<.001$, Cohen's $d=1.15$ ). Identical results were obtained when information about new treatment or surgery, done in the two years period between the baseline and the follow-up assessment, were included in the multiple regression.

\section{Changes over time}

Given the somewhat surprising findings demonstrating poor associations between ABS and multi-domain trait groups characterized at baseline with two-years follow-up measures, a contingency table (Table 4) was used to explore the proportion of individuals in the same or differing trait groups. Specifically, participant classifications by ABS and multi-domain trait groups were compared at baseline and the two-year time point. As reported in Table 4 (top), a proportion of participants varying from $42.9 \%$ to $64.6 \%$ remained in the respective ABS groups two years following the first assessment, with the larger change demonstrated in the Depressive/Reactive group, where $57.1 \%$ of the participants were classified in a different group (low or healthy) at follow-up assessment. The multidomain-trait groups showed a more consistent pattern across time, possibly explaining the reason the clinical pain measures were still associated with these groups in our longitudinal analyses. In fact, across the two multi-domain groups, only $\sim 30 \%$ of the participants changed in group status over time (Table 4 , bottom).

\begin{tabular}{|l|c|c|c|}
\hline ABS groups at baseline & \multicolumn{3}{|c|}{ ABS groups at two-years follow-up (\%) } \\
\hline & Healthy & Low & $\begin{array}{c}\text { Depressive/ } \\
\text { Reactive }\end{array}$ \\
\hline Healthy & $\mathbf{6 4 . 6}$ & 22.9 & 12.5 \\
\hline Low & 26.4 & $\mathbf{5 5 . 9}$ & 17.7 \\
\hline Depressive/Reactive & 14.2 & 42.9 & $\mathbf{4 2 . 9}$ \\
\hline Multi-domain traits at baseline & \multicolumn{3}{|c|}{$\begin{array}{c}\text { Multi-domain traits at two-years } \\
\text { follow-up (\%) }\end{array}$} \\
\hline & $\begin{array}{c}\text { Positive Valence } \\
\text { \& Cognition }\end{array}$ & $\begin{array}{c}\text { Negative Valence \& } \\
\text { Sensorimotor }\end{array}$ \\
\hline Positive Valence \& Cognition & $\mathbf{6 7 . 7}$ & 32.3 \\
\hline $\begin{array}{l}\text { Negative Valence \& } \\
\text { Sensorimotor }\end{array}$ & 26.7 & $\mathbf{7 3 . 3}$ \\
\hline
\end{tabular}

Table 4. Contingency table reporting in bold the proportion of participant (percentage) that remained stable in the respective groups of $A B S$ and multi-domain traits, and in gray the proportion of participants that moved to other groups.

\section{Discussion}

The overall aim of the current study was to evaluate the associations between dispositional traits conceptualized as ABS and multi-domain trait groups on health outcomes in a sample of middle aged and older adults with knee pain. Both groups showed that individuals in the vulnerable trait groups, depressive/reactive ABS group and negative valence and sensorimotor multi-trait group, 
reported higher levels of psychosocial distress and clinical pain. Interestingly, the pattern of findings was not fully replicated in the longitudinal analyses at the two-year follow-up. Only the multi-domain trait groups were predictive of clinical pain measures. Post-hoc exploratory analyses suggest that the lack of associations between ABS groups and outcomes of interest over time is the change in PA and NA, resulting in approximately a 50\% change in group membership across the entire sample, while multidomain groups remained relatively more stable with approximately $30 \%$ change.

Associations between ABS and pain-related outcomes at baseline. The current data confirm and extend the results of earlier studies in which ABS was investigated specific to clinical pain, functioning, and psychosocial outcomes, in individuals with fibromyalgia [27, 28], chronic low back pain [27], and healthy adults [19]. In line with previous studies, we found at baseline, that individuals with knee pain in the Depressive/Reactive ABS group had greater pain severity and depressive symptomatology than individuals in the Healthy or Low ABS groups. A number of psychological factors have been identified as predictive of clinical pain experiences such as pain catastrophizing, fear avoidance, and negative affect [53-58]. Underlying these psychological factors are neurobiologically based higher order predisposing patterns of functioning $[5,13]$. In individuals without chronic pain, Sibille et al. [19] showed that those with depressive ABS reported more somatic symptoms and higher levels of physical stimuli sensitivity. Combined with additional findings in chronic pain populations including those described here, dispositional traits provide an overarching conceptualization to encompass a vast array of psychological, physiological, and behavioral pain-related predictive factors [19, 27, 28]. Additionally, as dispositional traits are not pathologically-based, they provide the opportunity to communicate about an individual's functional style highlighting strengths as well as identifying vulnerabilities to target to boost resilience [59].

Associations between multi-domain traits and pain-related outcomes at baseline. Individuals in the Negative Valence and Sensorimotor group, compared to the individuals in the Positive Valence and Cognition group, reported greater pain severity and depressive symptomatology. Despite the wider range of associations demonstrated by a comprehensive multi-domain trait approach $[2,5,7]$, informed by RDoC domains, these groups shared similar profiles with ABS. The multi-domain trait groups were similar to the Healthy ABS group and the Depressive/Reactive ABS group. Among the additional domains, cognition is interesting to consider. Despite being historically thought in opposition to emotion, years of research in primates $[60]$ and humans $[61,62]$ convincingly showed that emotion and cognition are not dissociated modular systems but, instead, are highly integrated and interactive. The most paradigmatic example of this integration is represented by the amygdala, a region recognized among the major components of the emotional brain or the 'fear center' [63]. Despite its known role in emotional perception, especially in emotional visual processing [64-67], a consistent body of research has linked the amygdala to many functions classically associated with cognitive control, such as attention and decision making $[68,69]$. Here, individuals with high positive emotionality and low negative emotionality were further characterized by higher cognitive functioning than the other group. Recently, resilience was associated with amygdala volume in the same UPLOAD2 study [70].

Psychological interventions that incorporate positive affect alongside negative affect have already been proven to be more effective than the interventions focusing exclusively on negative affect [71-75]. Better understanding of which aspects of cognitive functioning are associated with dispositional traits might help inform the development of more effective clinical targets.

Associations between ABS and multi-domain traits, and pain-related outcomes at two-years. No associations were found between ABS and outcomes of interest at two-years after accounting for 
relevant covariates and baseline measures. Although the pattern observed at baseline was not fully replicated in the longitudinal analyses, multi-domain traits showed a significant association with clinical pain measures. Individuals in the negative valence and sensorimotor group reported higher pain severity than the individuals in the positive valence and cognition group. Post hoc analyses using contingency tables revealed that a consistent proportion of participants classified in the vulnerable ABS groups (Depressive/Reactive) at baseline changed over time, explaining the lack of associations between ABS groups and outcomes of interest in our longitudinal analyses. Across the entire sample, approximately $50 \%$ of the participants changed in group membership defined by ABS over two years. On the other hand, a smaller proportion of individuals changed multi-trait group classification ( 30\%) which might have contributed to the association between multi-domain trait groups and clinical pain. The finding supports the hypothesis that a comprehensive conceptualization of dispositional traits appears to be more informative than a measure limited to the affective domain of functioning [5]. From that perspective, the RDoC framework could represent a potentially useful approach for future research in dispositional trait characteristics in pain research.

Whether dispositions were defined using ABS or multi-domain traits, a proportion of individuals ( $\sim 30 \%$ to $\sim 50 \%$ ) changed in group membership classification over the two-year period. Changes of this magnitude are consistent with the extensive literature investigating the longitudinal trajectories of temperament in children and personality development. Caspi et al. [6] found that approximately 50\% of the individuals followed from childhood to young adulthood maintained consistent dispositional trait patterns contributing to stable personality characteristics over 20 years. Similar proportions have been reported in studies estimating that $30 \%-50 \%$ of the children who show consistently elevated negative affect develop diagnosable psychopathology later in life $[76,77]$. While these findings are generally interpreted as reliable indications that certain dispositional traits are associated with risk for psychopathology, they also led to the perception of trait-related stability rather than considering that at least $50 \%$ of the participants across all of these studies were also subject to change in their dispositional trait patterns. In addition, there is considerable evidence demonstrating that a stable personality is attained late in life [78] and that dispositional traits can be modified with trainings [79]. Since brain plasticity can be targeted to promote resilience and improve health outcomes [80], our findings are encouraging. Importantly, as dispositional traits tend to be fairly stable but are modifiable through experience and learning, understanding the factors facilitating changes are of great clinical importance.

Limitations. There are a few limitations in the study to consider. First, participants in the study are community dwelling adults with mild to moderate knee pain and do not necessarily represent individuals living with persisting and debilitating chronic pain. Second, although we include a covariate for chronic pain severity (total number of pain sites) in the analyses, the clinical pain measures in the study are specific to the knee and do not take in consideration the pain symptoms in other areas of the body. Third, we made a first attempt toward developing a more comprehensive dispositional trait measure informed by the RDoC matrix with a few adjustments. For example, the state PANAS is included among the self-report measures to investigate RDoC positive and negative valence. Trait PANAS, in addition to high correlation with the state measure, captures more of the temperamental feature, and studies have demonstrated utility in chronic pain [18]. In the sensorimotor domain, we included two sensory-related measures the PainDetect and warmth detection as a proxies. Although a temperament characteristic $[5,13]$, there is limited alignment with the sensorimotor dynamic sub-construct in the RDoC matrix. Whether or not sensory processing should be considered as a separate domain in the RDoC framework is debated [81]. Fourth, the lack of finding for experimental pain sensitivity may be the 
nature of measures investigated. More sustained measures of pain such as ischemic or cold pressor might be more informative [19]. Finally, the current investigations did consider possible treatment intervention differences over the two-year period but does not address other factors such as stress, pain, and sleep between the individuals whose dispositional trait group classifications remained the same and those whose classifications changed.

Summary. The current study evaluated the utility of dispositional trait measures, defined by ABS and multi-domain traits, for predicting clinical pain, psychosocial functioning, and experimental pain, in prospective analyses of adults with knee pain with or at risk for osteoarthritis. Despite a similar pattern of results between ABS and multi-domain traits observed at baseline, longitudinal analyses revealed only multi-domain trait groups defined at baseline were associated with clinical pain over time, suggesting that a multi-domain trait conceptualization is potentially a more informative operationalization of dispositional trait characteristics. Importantly, individuals with vulnerable traits (e.g., depressive/reactive ABS group) showed more variability across a variety of outcomes of interest at two years, suggesting that a vulnerable trait may represent a predisposition but not predetermining factor regarding pain-related functioning. Further research is needed to better understand factors contributing to improved outcomes in those with vulnerable dispositional traits, and potentially include additional biological and system-level measures. 


\section{References}

1. Posner, J., J.A. Russell, and B.S. Peterson, The circumplex model of affect: an integrative approach to affective neuroscience, cognitive development, and psychopathology. Dev Psychopathol, 2005. 17(3): p. 715-34.

2. Rettew, D.C. and L. McKee, Temperament and its role in developmental psychopathology. Harvard review of psychiatry, 2005. 13(1): p. 14-27.

3. Kroenke, K. and J. Unutzer, Closing the False Divide: Sustainable Approaches to Integrating Mental Health Services into Primary Care. J Gen Intern Med, 2017. 32(4): p. 404-410.

4. Kroenke, K., et al., Reciprocal relationship between pain and depression: a 12-month longitudinal analysis in primary care. J Pain, 2011. 12(9): p. 964-73.

5. Chess, S. and A. Thomas, Continuities and discontinuities in temperament, in Straight and devious pathways from childhood to adulthood. 1990, Cambridge University Press: New York, NY, US. p. 205-220.

6. Caspi, A., et al., Children's Behavioral Styles at Age 3 Are Linked to Their Adult Personality Traits at Age 26. Journal of Personality, 2003. 71(4): p. 495-513.

7. Trofimova, I. and W. Sulis, There is more to mental illness than negative affect: comprehensive temperament profiles in depression and generalized anxiety. BMC Psychiatry, 2018. 18(1): $p$. 125.

8. Watson, D., L.A. Clark, and A. Tellegen, Development and validation of brief measures of positive and negative affect: The PANAS scales. Journal of Personality and Social Psychology, 1988. 54(6): p. 1063-1070.

9. Lansade, L., M.F. Bouissou, and X. Boivin, Temperament in preweanling horses: development of reactions to humans and novelty, and startle responses. Dev Psychobiol, 2007. 49(5): p. 501-13.

10. Rothbart, M.K., S.A. Ahadi, and D.E. Evans, Temperament and personality: origins and outcomes. J Pers Soc Psychol, 2000. 78(1): p. 122-35.

11. Hur, J., et al., Dispositional negativity, cognition, and anxiety disorders: An integrative translational neuroscience framework. Prog Brain Res, 2019. 247: p. 375-436.

12. Shackman, A.J., et al., Dispositional negativity: An integrative psychological and neurobiological perspective. Psychol Bull, 2016. 142(12): p. 1275-1314.

13. Strelau, J., The regulative theory of temperament: current status. Personality and Individual Differences, 1996. 20(2): p. 131-142.

14. Robinson, D.L., How brain arousal systems determine different temperament types and the major dimensions of personality. Personality and Individual Differences, 2001. 31(8): p. 12331259.

15. Lee, L.O., et al., Optimism is associated with exceptional longevity in 2 epidemiologic cohorts of men and women. Proc Natl Acad Sci U S A, 2019. 116(37): p. 18357-18362.

16. Rettew, D.C. and L. McKee, Temperament and its role in developmental psychopathology. Harv Rev Psychiatry, 2005. 13(1): p. 14-27.

17. Sutin, A.R., et al., Personality traits and chronic disease: implications for adult personality development. The journals of gerontology. Series B, Psychological sciences and social sciences, 2013. 68(6): p. 912-920.

18. Hassett, A.L., et al., Affect and Low Back Pain: More to Consider Than the Influence of Negative Affect Alone. Clin J Pain, 2016. 32(10): p. 907-14.

19. Sibille, K.T., et al., Affect balance style, experimental pain sensitivity, and pain-related responses. Clin J Pain, 2012. 28(5): p. 410-7.

20. Patrick, C.J., J.J. Curtin, and A. Tellegen, Development and validation of a brief form of the Multidimensional Personality Questionnaire. Psychol Assess, 2002. 14(2): p. 150-63. 
21. Tellegen, A., Structures of mood and personality and their relevance to assessing anxiety, with an emphasis on self-report, in Anxiety and the anxiety disorders. 1985, Lawrence Erlbaum Associates, Inc: Hillsdale, NJ, US. p. 681-706.

22. Lang, P.J., The emotion probe. Studies of motivation and attention. Am Psychol, 1995. 50(5): $p$. 372-85.

23. Watson, D., et al., The two general activation systems of affect: Structural findings, evolutionary considerations, and psychobiological evidence. Journal of Personality and Social Psychology, 1999. 76(5): p. 820-838.

24. Derryberry, D. and M.A. Reed, Temperament and attention: orienting toward and away from positive and negative signals. J Pers Soc Psychol, 1994. 66(6): p. 1128-39.

25. Finan, P.H. and E.L. Garland, The role of positive affect in pain and its treatment. Clin J Pain, 2015. 31(2): p. 177-87.

26. Ong, A.D., et al., Positive affect and chronic pain: a preregistered systematic review and metaanalysis. Pain, 2020. 161(6): p. 1140-1149.

27. Hassett, A.L., et al., The relationship between affect balance style and clinical outcomes in fibromyalgia. Arthritis Rheum, 2008. 59(6): p. 833-40.

28. Toussaint, L.L., et al., A Comparison of Fibromyalgia Symptoms in Patients with Healthy versus Depressive, Low and Reactive Affect Balance Styles. Scand J Pain, 2014. 5(3): p. 161-166.

29. Berryman, C., et al., Evidence for working memory deficits in chronic pain: a systematic review and meta-analysis. Pain, 2013. 154(8): p. 1181-96.

30. Landrø, N.I., et al., The extent of neurocognitive dysfunction in a multidisciplinary pain centre population. Is there a relation between reported and tested neuropsychological functioning? Pain, 2013. 154(7): p. 972-7.

31. Moriarty, O. and D.P. Finn, Cognition and pain. Curr Opin Support Palliat Care, 2014. 8(2): p. 130-6.

32. Moriarty, O., B.E. McGuire, and D.P. Finn, The effect of pain on cognitive function: A review of clinical and preclinical research. Progress in Neurobiology, 2011. 93(3): p. 385-404.

33. Oosterman, J., et al., Executive and attentional functions in chronic pain: does performance decrease with increasing task load? Pain Res Manag, 2012. 17(3): p. 159-65.

34. Melzack R, C.K., Sensory, motivation and central control determinants of pain: a new conceptual model. The skin senses ed. K. D. 1968, Springfield, Illinois: Charles C. Thomas.

35. Dua, A.B., et al., Somatosensation in OA: exploring the relationships of pain sensitization, vibratory perception and spontaneous pain. BMC Musculoskeletal Disorders, 2018. 19(1): p. 307.

36. Cuthbert, B.N., The RDoC framework: facilitating transition from ICD/DSM to dimensional approaches that integrate neuroscience and psychopathology. World Psychiatry, 2014. 13(1): p. 28-35.

37. Cuthbert, B.N., Research Domain Criteria: toward future psychiatric nosologies. Dialogues Clin Neurosci, 2015. 17(1): p. 89-97.

38. Insel, T.R. and B.N. Cuthbert, Medicine. Brain disorders? Precisely. Science, 2015. 348(6234): p. 499-500.

39. Sibille, K.T., et al., Allostatic load and pain severity in older adults: Results from the English Longitudinal Study of Ageing. Experimental gerontology, 2017. 88: p. 51-58.

40. von Elm E, A.D., Egger M, Pocock SJ, Gøtzsche PC, Vandenbroucke JP, The Strengthening the Reporting of Observational Studies in Epidemiology (STROBE) statement: guidelines for reporting observational studies. J Clin Epidemiol, 2008. 61(4): p. 344-9.

41. Bartley, E.J., et al., Race/Ethnicity Moderates the Association Between Psychosocial Resilience and Movement-Evoked Pain in Knee Osteoarthritis. ACR Open Rheumatology, 2019. 1(1): p. 1625. 
42. Roux, C.H., et al., Screening for hip and knee osteoarthritis in the general population: predictive value of a questionnaire and prevalence estimates. Ann Rheum Dis, 2008. 67(10): p. 1406-11.

43. NIMH. Research Domain Criteria Initiative. RDoC Matrix 2009 [cited 2021; Available from: https://www.nimh.nih.gov/research/research-funded-by-nimh/rdoc/constructs/rdocmatrix.shtml.

44. Zimet, G.D., et al., The Multidimensional Scale of Perceived Social Support. Journal of Personality Assessment, 1988. 52(1): p. 30-41.

45. Schalet, B.D., et al., Clinical validity of PROMIS Depression, Anxiety, and Anger across diverse clinical samples. Journal of clinical epidemiology, 2016. 73: p. 119-127.

46. Bellamy, N., et al., Validation study of WOMAC: a health status instrument for measuring clinically important patient relevant outcomes to antirheumatic drug therapy in patients with osteoarthritis of the hip or knee. J Rheumatol, 1988. 15(12): p. 1833-40.

47. Bellamy, N. and W.W. Buchanan, A preliminary evaluation of the dimensionality and clinical importance of pain and disability in osteoarthritis of the hip and knee. Clin Rheumatol, 1986. 5(2): p. 231-41.

48. Von Korff, M., et al., Grading the severity of chronic pain. Pain, 1992. 50(2): p. 133-149.

49. Guralnik, J.M., et al., A short physical performance battery assessing lower extremity function: association with self-reported disability and prediction of mortality and nursing home admission. J Gerontol, 1994. 49(2): p. M85-94.

50. Guralnik, J.M., et al., Lower-extremity function in persons over the age of 70 years as a predictor of subsequent disability. N Engl J Med, 1995. 332(9): p. 556-61.

51. Ding, C. and X. He, Linearized cluster assignment via spectral ordering, in Proceedings of the twenty-first international conference on Machine learning. 2004, Association for Computing Machinery: Banff, Alberta, Canada. p. 30.

52. Rousseeuw, P.J., Silhouettes: A graphical aid to the interpretation and validation of cluster analysis. Journal of Computational and Applied Mathematics, 1987. 20: p. 53-65.

53. Wong, W.S., et al., The fear-avoidance model of chronic pain: assessing the role of neuroticism and negative affect in pain catastrophizing using structural equation modeling. Int J Behav Med, 2015. 22(1): p. 118-31.

54. Vachon-Presseau, E., et al., The Emotional Brain as a Predictor and Amplifier of Chronic Pain. J Dent Res, 2016. 95(6): p. 605-12.

55. Ruiz-Aranda, D., J.M. Salguero, and P. Fernández-Berrocal, Emotional intelligence and acute pain: the mediating effect of negative affect. J Pain, 2011. 12(11): p. 1190-6.

56. Mounce, C., E. Keogh, and C. Eccleston, A principal components analysis of negative affectrelated constructs relevant to pain: evidence for a three component structure. J Pain, 2010. 11(8): p. 710-7.

57. Jones, D.A., et al., The relationship between cognitive appraisal, affect, and catastrophizing in patients with chronic pain. J Pain, 2003. 4(5): p. 267-77.

58. Quartana, P.J., C.M. Campbell, and R.R. Edwards, Pain catastrophizing: a critical review. Expert Rev Neurother, 2009. 9(5): p. 745-58.

59. Stieger, M., et al., Changing personality traits with the help of a digital personality change intervention. Proc Natl Acad Sci U S A, 2021. 118(8).

60. Goldman-Rakic, P.S., Topography of cognition: parallel distributed networks in primate association cortex. Annu Rev Neurosci, 1988. 11: p. 137-56.

61. Pessoa, L., On the relationship between emotion and cognition. Nat Rev Neurosci, 2008. 9(2): $p$. 148-58. 
62. Pessoa, L., The cognitive-emotional brain: From interactions to integration. The cognitiveemotional brain: From interactions to integration. 2013, Cambridge, MA, US: MIT Press. xii, 320xii, 320.

63. Ohman, A. and S. Mineka, Fears, phobias, and preparedness: toward an evolved module of fear and fear learning. Psychol Rev, 2001. 108(3): p. 483-522.

64. Sabatinelli, D., et al., Parallel amygdala and inferotemporal activation reflect emotional intensity and fear relevance. Neuroimage, 2005. 24(4): p. 1265-70.

65. Sabatinelli, D., et al., Emotional perception: meta-analyses of face and natural scene processing. Neuroimage, 2011. 54(3): p. 2524-33.

66. Sambuco, N., et al., Transdiagnostic trauma severity in anxiety and mood disorders: Functional brain activity during emotional scene processing. Psychophysiology, 2020. 57(1): p. e13349.

67. Sambuco, N., et al., Aversive perception in a threat context: Separate and independent neural activation. Biol Psychol, 2020. 154: p. 107926.

68. Gupta, R., et al., The amygdala and decision-making. Neuropsychologia, 2011. 49(4): p. 760-6.

69. McNally, G.P., J.P. Johansen, and H.T. Blair, Placing prediction into the fear circuit. Trends Neurosci, 2011. 34(6): p. 283-92.

70. Tanner, J.J., et al., Resilience, pain, and the brain: Relationships differ by sociodemographics. J Neurosci Res, 2021.

71. Bolier, L., et al., Positive psychology interventions: a meta-analysis of randomized controlled studies. BMC Public Health, 2013. 13: p. 119.

72. Fredrickson, B.L., The role of positive emotions in positive psychology. The broaden-and-build theory of positive emotions. Am Psychol, 2001. 56(3): p. 218-26.

73. Schore, A.N., Back to basics: attachment, affect regulation, and the developing right brain: linking developmental neuroscience to pediatrics. Pediatr Rev, 2005. 26(6): p. 204-17.

74. Sin, N.L. and S. Lyubomirsky, Enhancing well-being and alleviating depressive symptoms with positive psychology interventions: a practice-friendly meta-analysis. J Clin Psychol, 2009. 65(5): p. 467-87.

75. Hemington, K.S., et al., Beyond Negative Pain-Related Psychological Factors: Resilience Is Related to Lower Pain Affect in Healthy Adults. J Pain, 2017. 18(9): p. 1117-1128.

76. Clauss, J.A. and J.U. Blackford, Behavioral inhibition and risk for developing social anxiety disorder: a meta-analytic study. J Am Acad Child Adolesc Psychiatry, 2012. 51(10): p. 10661075.e1.

77. Hengartner, M.P., et al., Relationship between personality and psychopathology in a longitudinal community study: a test of the predisposition model. Psychol Med, 2016. 46(8): p. 1693-705.

78. Roberts, B.W. and W.F. DelVecchio, The rank-order consistency of personality traits from childhood to old age: A quantitative review of longitudinal studies. Psychological Bulletin, 2000. 126(1): p. 3-25.

79. Stieger, M., et al., Changing personality traits with the help of a digital personality change intervention. Proceedings of the National Academy of Sciences, 2021. 118(8): p. e2017548118.

80. Hanssen, M.M., et al., Can positive affect attenuate (persistent) pain? State of the art and clinical implications. Curr Rheumatol Rep, 2017. 19(12): p. 80.

81. Harrison, L.A., et al., The Importance of Sensory Processing in Mental Health: A Proposed Addition to the Research Domain Criteria (RDoC) and Suggestions for RDoC 2.0. Front Psychol, 2019. 10: p. 103. 


\section{SUPPLEMENTARY APPENDIX}

Vulnerable Dispositional Traits and Chronic Pain: Predisposing but not Predetermining

\section{Contents}

Table S1. Measures used in the principal component analysis (PCA) ................................................ 22

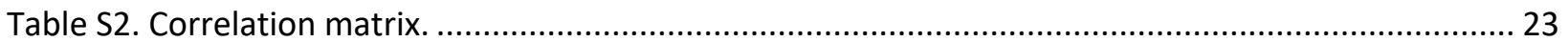

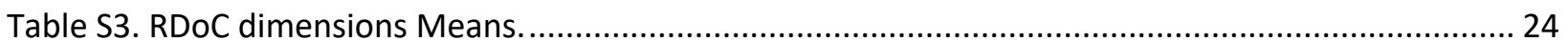




\section{Table S1. Measures used in the principal}

component analysis (PCA). In bold, the highest loadings for each of the five RDoC dimensions of negative valence system, arousal/regulatory system, cognitive system, positive valence system, sensorimotor system. Highest loadings (> .60) are reported in bold.

\begin{tabular}{|l|c|c|c|c|c|}
\hline \multirow{2}{*}{ Measures } & \multicolumn{5}{|c|}{ Principal Component Analysis } \\
\hline & $\begin{array}{c}\text { Negative } \\
\text { Valence } \\
\text { System }\end{array}$ & $\begin{array}{c}\text { Arousal / } \\
\text { Regulatory } \\
\text { System }\end{array}$ & $\begin{array}{c}\text { Cognitive } \\
\text { System }\end{array}$ & $\begin{array}{c}\text { Positive } \\
\text { Valence } \\
\text { System }\end{array}$ & $\begin{array}{c}\text { Sensorimo } \\
\text { tor System }\end{array}$ \\
\hline PANAS-PA & .01 & -.09 & -.08 & .89 & -.02 \\
\hline LOT-R (optimism) & -.16 & .02 & .20 & .71 & -.03 \\
\hline PANAS-NA & .71 & -.09 & -.08 & -.25 & -.01 \\
\hline PROMIS-Anxiety & .80 & -.14 & .11 & -.14 & -.02 \\
\hline MoCA Total Score & -.15 & .06 & .87 & -.09 & -.02 \\
\hline REALM-R & .18 & -.04 & .87 & .15 & .04 \\
\hline FSS & .75 & .05 & .13 & -.01 & -.05 \\
\hline Systolic Blood Pressure & -.04 & .91 & .01 & -.01 & -.02 \\
\hline $\begin{array}{l}\text { Diastolic Blood } \\
\text { Pressure }\end{array}$ & .04 & .91 & .03 & -.07 & .01 \\
\hline PD-Q & .68 & .18 & -.26 & .16 & .09 \\
\hline $\begin{array}{l}\text { Warmth Detection- } \\
\text { arm }\end{array}$ & -.03 & .07 & -.02 & .03 & .79 \\
\hline $\begin{array}{l}\text { Warmth Detection- } \\
\text { knee }\end{array}$ & -.01 & -.08 & .05 & -.06 & .82 \\
\hline PANAS-PA: Posive & & & & \\
\hline
\end{tabular}

PANAS-PA: Positive and Negative Affect Schedule Positive Affect, LOT-R: Life Orientation Test-Revised, PANAS-NA: Positive and Negative Affect Schedule Negative Affect, MoCA: Montreal Cognitive Assessment, REALM-R: Rapid Estimate of Adult Literacy in Medicine, Revised, FSS: Fatigue Severity Scale, SPB: systolic blood pressure, DBP: diastolic blood pressure, PD-Q: painDETECT 


\section{Table S2. Correlation matrix. correlation matrix}

illustrating the correlation pattern (Pearson $r$ ) between each pair of factors resulting from the principal component analysis (PCA).

\begin{tabular}{|l|c|c|c|c|c|}
\hline & $\begin{array}{c}\text { Negative } \\
\text { Valence } \\
\text { System }\end{array}$ & $\begin{array}{c}\text { Arousal / } \\
\text { Regulatory } \\
\text { System }\end{array}$ & $\begin{array}{c}\text { Cognitive } \\
\text { System }\end{array}$ & $\begin{array}{c}\text { Positive } \\
\text { Valence } \\
\text { System }\end{array}$ & $\begin{array}{c}\text { Sensorimotor } \\
\text { System }\end{array}$ \\
\hline $\begin{array}{l}\text { Negative } \\
\text { Valence } \\
\text { System }\end{array}$ & - & - & & & \\
\hline $\begin{array}{l}\text { Arousal / } \\
\text { Regulatory } \\
\text { System }\end{array}$ & .03 & -.14 & - & & \\
\hline $\begin{array}{l}\text { Cognitive } \\
\text { System }\end{array}$ & -.14 & .04 & .07 & - & \\
\hline $\begin{array}{l}\text { Positive } \\
\text { Valence } \\
\text { System }\end{array}$ & -.30 & .11 & -.09 & .07 & \\
\hline $\begin{array}{l}\text { Sensorimotor } \\
\text { System }\end{array}$ & .06 & & & & \\
\hline
\end{tabular}




\section{Table S3. RDoC dimensions Means. Means and maximum to}

minimum score for each of the variables included in the Principal Component Analysis (PCA) used to identify the RDoC dimensions. Group comparison reports the unadjusted $p$-value.

\begin{tabular}{|l|c|c|c|c|}
\hline Variable & \multicolumn{1}{|c|}{$\begin{array}{c}\text { Total } \\
(\mathbf{N}=\mathbf{1 6 7})\end{array}$} & $\begin{array}{c}\text { Positive Valence and } \\
\text { Cognition } \\
\mathbf{( N = 1 0 6 )}\end{array}$ & $\begin{array}{c}\text { Negative Valence and } \\
\text { Sensorimotor } \\
\text { (N=61) }\end{array}$ & $\begin{array}{c}\text { Group } \\
\text { Comparison } \\
\text { P-value }\end{array}$ \\
\hline PANAS-PA & $35[29.0$ to 40.0$]$ & $38.0[34.0$ to 42.0$]$ & $30.0[25.0$ to 37.0$]$ & $<0.0001$ \\
\hline LOT-R (optimism) & $18.0[14.0$ to 22.0$]$ & $21.0[17.0$ to 23.0$]$ & $14.0[11.0$ to 16.0$]$ & $<0.0001$ \\
\hline PANAS-NA & $15.0[11.5$ to 19.0$]$ & $13.0[11.0$ to 15.0$]$ & $21.0[17.0$ to 26.0$]$ & $<0.0001$ \\
\hline PROMIS-Anxiety & $51.3[44.7$ to 57.6$]$ & $46.7[42.1$ to 52.6$]$ & $56.3[51.3$ to 61.3$]$ & $<0.0001$ \\
\hline MoCA Total Score & $24.0[22.0$ to 27.0$]$ & $26.0[24.0$ to 28.0$]$ & $22.0[20.0$ to 24.0$]$ & $<0.0001$ \\
\hline REALM-R & $8.0[6.0$ to 8.0$]$ & $8.0[7.0$ to 8.0$]$ & $7.0[4.0$ to 8.0$]$ & $<0.0001$ \\
\hline FSS & $27.0[17.5$ to 39.0$]$ & $22.5[15.0$ to 32.0$]$ & $35.0[26.0$ to 46.0$]$ & $<0.0001$ \\
\hline SBP & $122.7[114.0$ to 136.0$]$ & $122.7[114.0$ to 136.7$]$ & $121.7[112.3$ to 135.7$]$ & 0.9814 \\
\hline DBP & $73.0[67.2$ to 79.5$]$ & $72.8[66.7$ to 78.3$]$ & $74.3[67.3$ to 80.0$]$ & 0.2897 \\
\hline Warmth Detection-arm & $34.7[34.0$ to 35.6$]$ & $34.4[33.9$ to 35.6$]$ & $34.9[34.2$ to 35.7$]$ & 0.0298 \\
\hline Warmth Detection-knee & $36.0[34.9$ to 39.7$]$ & $35.6[34.7$ to 37.4$]$ & $37.7[35.5$ to 41.0$]$ & 0.0020 \\
\hline
\end{tabular}

PANAS-PA: Positive and Negative Affect Schedule Positive Affect, LOT-R: Life Orientation Test-Revised, PANAS-NA: Positive and Negative Affect Schedule Negative Affect, MoCA: Montreal Cognitive Assessment, REALM-R: Rapid Estimate of Adult Literacy in Medicine, Revised, FSS: Fatigue Severity Scale, SPB: systolic blood pressure, DBP: diastolic blood pressure 